\title{
Transition from adiabatic inspiral to plunge into a spinning black hole
}

\author{
Michael Kesden* \\ Center for Cosmology and Particle Physics, Department of Physics, \\ New York University, 4 Washington Pl., New York, New York 10003, USA
}

(Dated: January 2011)

\begin{abstract}
A test particle of mass $\mu$ on a bound geodesic of a Kerr black hole of mass $M \gg \mu$ will slowly inspiral as gravitational radiation extracts energy and angular momentum from its orbit. This inspiral can be considered adiabatic when the orbital period is much shorter than the timescale on which energy is radiated, and quasicircular when the radial velocity is much less than the azimuthal velocity. Although the inspiral always remains adiabatic provided $\mu \ll M$, the quasicircular approximation breaks down as the particle approaches the innermost stable circular orbit (ISCO). In this paper, we relax the quasicircular approximation and solve the radial equation of motion explicitly near the ISCO. We use the requirement that the test particle's 4-velocity remains properly normalized to calculate a new contribution to the difference between its energy and angular momentum. This difference determines how a black hole's spin changes following a test-particle merger, and can be extrapolated to help predict the mass and spin of the final black hole produced in finite-mass-ratio black-hole mergers. Our new contribution is particularly important for nearly maximally spinning black holes, as it can affect whether a merger produces a naked singularity.
\end{abstract}

\section{INTRODUCTION}

Supermassive black holes (SBHs) with masses $10^{6} M_{\odot} \lesssim M \lesssim 10^{10} M_{\odot}$ reside at the centers of most large galaxies [1. These SBHs will be surrounded by dense cusps of stars 2, some fraction of which will consist of compact objects (white dwarfs, neutron stars, or stellar-mass black holes) of mass $\mu \sim 1-10 M_{\odot}$. Compact objects whose orbital velocities lie within a "loss cone" about the radial direction [3] will inspiral into the SBHs under the influence of gravitational radiation 4. These extreme-mass-ratio inspirals (EMRIs) will be an important source of gravitational waves (GWs) for the proposed space-based GW detector LISA [5. LISA will rely on matched filtering to detect EMRIs, requiring templates that accurately track the phase of the GWs when their fundamental frequency $f$ is in the range $10^{-4} \lesssim f \lesssim 10^{-1} \mathrm{~Hz}$. These GW frequencies correspond to orbital frequencies $\Omega=\pi f$ at the innermost stable circular orbits (ISCOs) of SBHs with masses $10^{5} M_{\odot} \lesssim M \lesssim 10^{7} M_{\odot}$. To maximize the number of EMRIs detected by LISA, it is therefore essential to understand the trajectories of compact objects as they inspiral all the way to the ISCO.

In the absence of gravitational radiation, test particles travel on geodesics of the Kerr metric $g_{\mu \nu}$ [6] that describes the spacetime of spinning black holes. Kerr geodesics are characterized by four constants of motion: the particle's rest mass $\mu$, energy $E, z$ component of angular momentum $L_{z}$, and Carter constant $Q$ [7]. These constants can be determined from the particle's 4-momentum $p^{\mu}$ and the Kerr metric's timelike Killing vector $T_{\mu}$, azimuthal Killing vector $\Phi_{\mu}$, and Killing ten-

*Electronic address: mhk10@nyu.edu sor $Q_{\mu \nu}[$ :

$$
\begin{aligned}
\mu & =\sqrt{-g_{\mu \nu} p^{\mu} p^{\nu}} \\
E & =-T_{\mu} p^{\mu} \\
L_{z} & =\Phi_{\mu} p^{\mu} \\
Q & =Q_{\mu \nu} p^{\mu} p^{\nu} .
\end{aligned}
$$

Equatorial geodesics have $Q=0$; for computational simplicity we will restrict our attention to equatorial orbits for the remainder of this paper.

In the test-particle limit $\eta \equiv \mu / M \ll 1$, the stressenergy tensor of a test particle moving on a Kerr geodesic sources gravitational radiation that can be calculated using black-hole perturbation theory [9]. This radiation extracts energy and angular momentum from the orbit, causing the particle to migrate through the phase space $\left\{E, L_{z}\right\}$. The GW energy flux $\dot{E}_{\mathrm{GW}}$ and angular momentum flux $\dot{L}_{z \mathrm{GW}}$ (an overdot symbolizes a derivative with respect to Boyer-Lindquist coordinate time) are proportional to $\eta^{2}$, implying that the timescale $t_{\mathrm{GW}} \sim E / \dot{E}_{\mathrm{GW}}$ is proportional to $\eta^{-1}$. The orbital period $t_{\text {orb }} \sim \Omega^{-1}$ is independent of $\eta$ to lowest order, implying that the inspiral will always be adiabatic $\left(t_{\mathrm{GW}} \gg t_{\mathrm{orb}}\right.$ ) for sufficiently small $\eta$. Gravitational radiation circularizes eccentric orbits in the post-Newtonian regime [10, implying that the early inspiral will also be quasicircular $(\dot{r} \ll r \Omega)$. During this adiabatic, quasicircular portion of the inspiral, the trajectory of the test particle is well described by a sequence of circular geodesics of ever decreasing BoyerLindquist radius $r$. In the quasicircular approximation, the radial velocity is given by

$$
\dot{r}=-\frac{\dot{E}_{\mathrm{GW}}}{d E / d r}
$$

where $d E / d r$ is the derivative of the energy of a circular equatorial geodesic with respect to its Boyer-Lindquist radius. This radial velocity is proportional to $\eta$, and 
approaches negative infinity as $r$ approaches $r_{\text {ISCO }}$ where $d E / d r$ vanishes. This behavior is unphysical, and reflects the breakdown of the quasicircular approximation as the test particle approaches the ISCO.

In the vicinity of the ISCO, the test particle transitions from the quasicircular inspiral described above to a "captured" plunge that crosses the event horizon [11. This transition regime has been investigated in the testparticle limit by Ori and Thorne [12] (hereafter OT) and for nonspinning black holes or arbitrary mass ratio by Buonanno and Damour [13. Sundararajan [14] generalized the approach of OT to inclined and eccentric orbits, deriving trajectories that served as sources for the gravitational waveforms calculated in Sundararajan, Khanna, and Hughes [15]. We will adopt the notation of OT throughout this paper to facilitate comparisons between our results and theirs. In Sec. II] we will review the previous treatment of the transition regime and the discovery of scaling relations that can be used to apply a universal dimensionless trajectory to mergers with arbitrary mass ratio and black-hole spin. In Sec. III], we show that the test particle's 4-momentum $p^{\mu}$ is not properly normalized according to Eq. 1al in this model of the transition. We develop a new model that satisfies this requirement by allowing the particle's energy and angular momentum to vary independently. We then explore this new model's predictions for the behavior of the transition regime for nearly maximal black-hole spins in Sec. IV] A summary of our principle findings and their implications is given in Sec. V

\section{PREVIOUS TREATMENT}

\section{A. Geodesic Motion}

We begin by reviewing the motion of test particles on Kerr geodesics. In the equatorial plane $(\theta=\pi / 2)$, the Kerr metric for a black hole of mass $M$ and spin $a$ can be written in Boyer-Lindquist coordinates [16] as

$$
\begin{aligned}
& d s^{2}=-\left(1-\frac{2 M}{r}\right) d t^{2}+\left(1-\frac{2 M}{r}+\frac{a^{2}}{r^{2}}\right)^{-1} d r^{2} \\
& +r^{2} d \theta^{2}+\left(r^{2}+a^{2}+\frac{2 M a^{2}}{r}\right) d \phi^{2}-\frac{4 M a}{r} d t d \phi
\end{aligned}
$$

Here and throughout this paper we use units in which Newton's constant $G$ and the speed of light $c$ are unity. We can also define a dimensionless radius $\tilde{r} \equiv r / M$, coordinate time $\tilde{t} \equiv t / M$, and spin $\tilde{a} \equiv a / M$. In these coordinates, the three constants of motion given in Eqs. (1a) through (1c) provide three equations for the evolution of $\tilde{t}, \tilde{r}$, and $\phi$ as functions of the dimensionless proper time $\tilde{\tau} \equiv \tau / M$ along the particle's worldline. Eq. 1a can be rewritten as

$$
\begin{aligned}
1= & -g_{\mu \nu} \frac{d \tilde{x}^{\mu}}{d \tilde{\tau}} \frac{d \tilde{x}^{\nu}}{d \tilde{\tau}} \\
= & \left(1-\frac{2}{\tilde{r}}\right)\left(\frac{d \tilde{t}}{d \tilde{\tau}}\right)^{2}-\left(1-\frac{2}{\tilde{r}}+\frac{\tilde{a}^{2}}{\tilde{r}^{2}}\right)^{-1}\left(\frac{d \tilde{r}}{d \tilde{\tau}}\right)^{2} \\
& -\left(\tilde{r}^{2}+\tilde{a}^{2}+\frac{2 \tilde{a}^{2}}{\tilde{r}}\right)\left(\frac{d \phi}{d \tilde{\tau}}\right)^{2}+\frac{4 \tilde{a}}{\tilde{r}} \frac{d \tilde{t}}{d \tilde{\tau}} \frac{d \phi}{d \tilde{\tau}}
\end{aligned}
$$

while according to Eqs. (1b) and (1c), the dimensionless energy $\tilde{E} \equiv E / \mu$, and angular momentum $\tilde{L} \equiv L_{z} / \mu M$ are given by

$$
\begin{aligned}
& \tilde{E}=\left(1-\frac{2}{\tilde{r}}\right) \frac{d \tilde{t}}{d \tilde{\tau}}+\frac{2 \tilde{a}}{\tilde{r}} \frac{d \phi}{d \tilde{\tau}} \\
& \tilde{L}=\left(\tilde{r}^{2}+\tilde{a}^{2}+\frac{2 \tilde{a}^{2}}{\tilde{r}}\right) \frac{d \phi}{d \tilde{\tau}}-\frac{2 \tilde{a}}{\tilde{r}} \frac{d \tilde{t}}{d \tilde{\tau}}
\end{aligned}
$$

Solving Eqs. (5a) and $(5 \mathrm{~b}$ for $d \tilde{t} / d \tilde{\tau}$ and $d \phi / d \tilde{\tau}$, inserting the result into Eq. 4, then solving for $(d \tilde{r} / d \tilde{\tau})^{2}$ yields

$$
\left(\frac{d \tilde{r}}{d \tilde{\tau}}\right)^{2}=\tilde{E}^{2}-V(\tilde{r}, \tilde{E}, \tilde{L})
$$

where

$$
V(\tilde{r}, \tilde{E}, \tilde{L}) \equiv 1-\frac{2}{\tilde{r}}+\frac{\tilde{L}^{2}+\tilde{a}^{2}-\tilde{E}^{2} \tilde{a}^{2}}{\tilde{r}^{2}}-\frac{2(\tilde{L}-\tilde{E} \tilde{a})^{2}}{\tilde{r}^{3}}
$$

is the effective potential.

Geodesic motion is alternatively described by the geodesic equations

$$
\frac{d^{2} \tilde{x}^{\mu}}{d \tilde{\tau}^{2}}+\Gamma_{\alpha \beta}^{\mu} \frac{d \tilde{x}^{\alpha}}{d \tilde{\tau}} \frac{d \tilde{x}^{\beta}}{d \tilde{\tau}}=0
$$

where $\Gamma_{\alpha \beta}^{\mu}$ are the Christoffel symbols for the Kerr metric. When $\tilde{E}$ and $\tilde{L}$ are constants, the second of these equations $\left(\tilde{x}^{1}=\tilde{r}\right)$ is equivalent to the derivative of Eq. 6. with respect to $\tilde{\tau}$

$$
\frac{d^{2} \tilde{r}}{d \tilde{\tau}^{2}}=-\frac{1}{2} \frac{\partial V}{\partial \tilde{r}}
$$

\section{B. Near the ISCO}

In the vicinity of the ISCO, Eq. (6) can be Taylor expanded about the ISCO values of $\tilde{r}, \tilde{E}$, and $\tilde{L}$. Expanding in the small variables

$$
\begin{aligned}
R & \equiv \tilde{r}-\tilde{r}_{\mathrm{ISCO}}, \\
\chi & \equiv \tilde{\Omega}_{\mathrm{ISCO}}^{-1}\left(\tilde{E}-\tilde{E}_{\mathrm{ISCO}}\right) \\
\xi & \equiv \tilde{L}-\tilde{L}_{\mathrm{ISCO}}
\end{aligned}
$$

where $\tilde{\Omega}_{\text {ISCO }}$ is the orbital frequency at the ISCO, yields

$$
\begin{aligned}
\left(\frac{d R}{d \tilde{\tau}}\right)^{2}= & -\frac{2 \alpha}{3} R^{3}+2 \beta R \xi+\frac{\partial V}{\partial \tilde{L}}(\chi-\xi) \\
& -\tilde{\Omega} \frac{\partial^{2} V}{\partial \tilde{E} \partial \tilde{r}}(\chi-\xi) R+\ldots
\end{aligned}
$$


where the ellipsis denotes higher-order terms. Following the OT notation we define

$$
\begin{aligned}
& \alpha \equiv \frac{1}{4}\left(\frac{\partial^{3} V}{\partial \tilde{r}^{3}}\right)_{\mathrm{ISCO}} \\
& \beta \equiv-\frac{1}{2}\left(\frac{\partial^{2} V}{\partial \tilde{L} \partial \tilde{r}}+\tilde{\Omega} \frac{\partial^{2} V}{\partial \tilde{E} \partial \tilde{r}}\right)_{\mathrm{ISCO}}
\end{aligned}
$$

and we have made use of the relation

$$
\tilde{E}-\frac{1}{2} \frac{\partial V}{\partial \tilde{E}}-\frac{1}{2} \tilde{\Omega}^{-1} \frac{\partial V}{\partial \tilde{L}}=0
$$

which holds at extrema of the effective potential. Again assuming that $\tilde{E}$ and $\tilde{L}$ are constant, Eq. 11 is equivalent to

$$
\frac{d^{2} R}{d \tilde{\tau}^{2}}=-\alpha R^{2}+\beta \xi-\frac{1}{2} \tilde{\Omega} \frac{\partial^{2} V}{\partial \tilde{E} \partial \tilde{r}}(\chi-\xi) .
$$

Terms of $\mathcal{O}\left(R^{3}\right), \mathcal{O}\left(\xi^{2}, \chi^{2}\right)$ and higher order have been neglected in Eq. 14.

\section{Radiation Reaction}

We have so far neglected the effects of radiation reaction on the test particle's motion. The proper way to include radiation reaction would be to calculate the conservative and dissipative parts of the self-force [17, and add these additional terms to the right-hand side of Eq. (8). Although self-force calculations have progressed rapidly in recent years (for recent reviews see [18, 19]), the full self-force is not yet available for test particles on Kerr geodesics near the ISCO. Without this self-force, the effects of radiation reaction can be approximated by making the energy and angular momentum time dependent in Eqs. (6) and (9).

For particles on circular orbits, the rates at which energy and angular momentum are radiated are related by

$$
\frac{d \tilde{E}}{d \tilde{\tau}}=\tilde{\Omega} \frac{d \tilde{L}}{d \tilde{\tau}} .
$$

OT assume that this relation holds throughout the transition, implying that

$$
\chi=\xi=-\eta \kappa \tilde{\tau}
$$

where

$$
\kappa \equiv\left(\tilde{\Omega}^{-1} \frac{d \tilde{E}}{d \tilde{t}} \frac{d \tilde{t}}{d \tilde{\tau}}\right)_{\mathrm{ISCO}}
$$

We will later relax this assumption in Sec. III. Inserting Eq. 16 into Eq. 14 yields

$$
\frac{d^{2} R}{d \tilde{\tau}^{2}}=-\alpha R^{2}-\eta \beta \kappa \tilde{\tau},
$$

identical to Eq. (3.15) of OT.

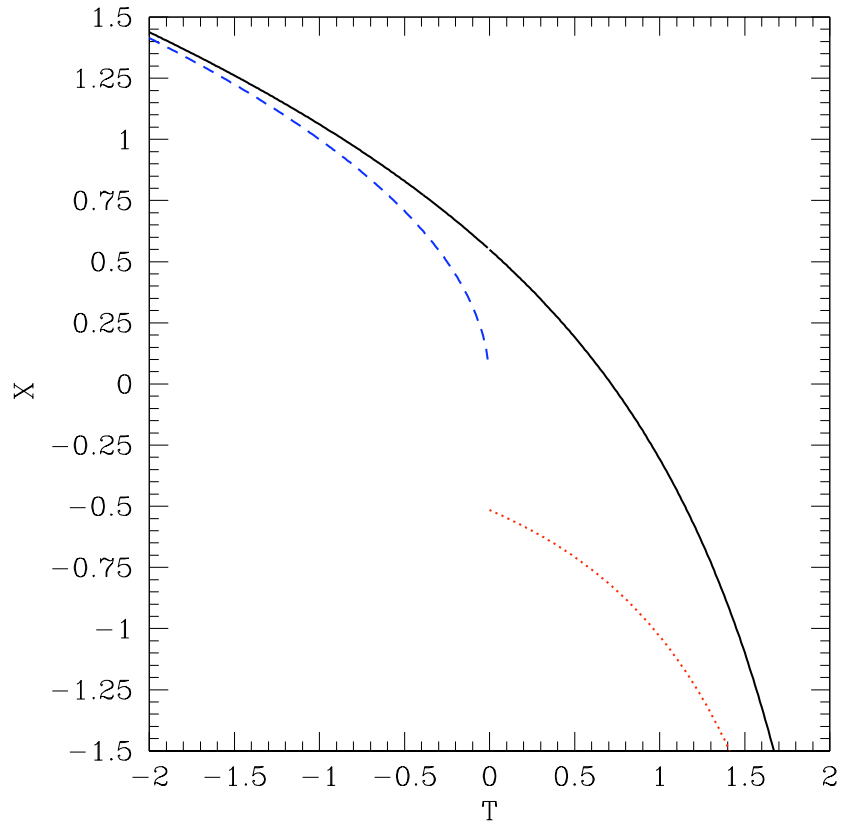

FIG. 1: The dimensionless radius $X$ as a function of dimensionless time $T$ during the transition from adiabatic inspiral to plunge. The solid black curve shows the numerical solution to Eq. 21, while the dashed blue and dotted red curves show the approximate analytic solutions at early and late times given by Eqs. 22 and 23 respectively. This figure is a reproduction of Fig. 2 in $\mathrm{OT}$.

\section{Dimensionless Equation of Motion}

Previous studies [12, 13] noticed that Eq. (18) can be converted into dimensionless form by defining

$$
\begin{aligned}
R & \equiv \eta^{2 / 5} R_{0} X, \\
\tilde{\tau} & \equiv \eta^{-1 / 5} \tau_{0} T,
\end{aligned}
$$

where

$$
\begin{aligned}
R_{0} & =(\beta \kappa)^{2 / 5} \alpha^{-3 / 5} \\
\tau_{0} & =(\alpha \beta \kappa)^{-1 / 5}
\end{aligned}
$$

In these variables, Eq. 18 becomes

$$
\frac{d^{2} X}{d T^{2}}=-X^{2}-T
$$

At early times, the particle's radial velocity and acceleration approach zero, suggesting that the correct solution to Eq. 21 asymptotes to

$$
X=\sqrt{-T} \quad T \rightarrow-\infty .
$$

At late times, the particle plunges into the horizon $(X=$ $-\infty)$ in a finite proper time $\tilde{\tau}$. The second term on the 
right-hand of Eq. 21] can then be neglected, yielding the approximate solution

$$
X=\frac{-6}{\left(T_{\text {plunge }}-T\right)^{2}} \quad T \rightarrow T_{\text {plunge }} .
$$

Numerically, $T_{\text {plunge }}=3.412$. The numerical solution to Eq. (21) and approximate analytic solutions of Eqs. 220 and (23) are given by the solid black, dashed blue, and dotted red curves in Fig. 1 .

In addition to the approximate radial trajectory $X(T)$, this model also provides an estimate of the energy and angular momentum radiated during the transition. Assuming that energy and angular momentum are radiated at the ISCO rate throughout the transition, Eq. (23) implies that the test particle will radiate an additional amount

$$
\begin{aligned}
\Delta \tilde{E}_{\mathrm{tr}} & \equiv \tilde{E}_{\mathrm{ISCO}}-\tilde{E}_{\mathrm{final}}=\eta^{4 / 5} \tilde{\Omega}_{\mathrm{ISCO}} \kappa \tau_{0} T_{\text {plunge }} \\
\Delta \tilde{L}_{\mathrm{tr}} & \equiv \tilde{L}_{\mathrm{ISCO}}-\tilde{L}_{\text {final }}=\eta^{4 / 5} \kappa \tau_{0} T_{\text {plunge }}
\end{aligned}
$$

beyond that calculated in the adiabatic approximation. Since $\Delta \tilde{E}_{\mathrm{tr}}$ and $\Delta \tilde{L}_{\mathrm{tr}}$ scale as $\eta^{4 / 5}$, in the test-particle limit $\eta \rightarrow 0$ the specific energy $\tilde{E}$ and angular momentum $\tilde{L}$ radiated during the transition should exceed that radiated during the ringdown which scales as $\eta$. Given the success of efforts to predict black-hole spins by extrapolating from the test-particle limit $20-23$, a closer examination of the energy and angular momentum radiated during the transition is worthwhile.

\section{NORMALIZATION OF 4-VELOCITY}

We noted in Sec. II A that the normalization of the 4velocity (4) and the geodesic equation (8) provide alternative equations of radial motion. While they are equivalent for geodesics, they differ once $\tilde{E}$ and $\tilde{L}$ become time dependent. Ori and Thorne [12] solve the geodesic equation under the assumption that $\tilde{E}$ and $\tilde{L}$ vary with proper time according to Eq. (16). What does this assumption imply for the norm of the 4-velocity? Expressed in the dimensionless variables of Eq. (19), Eq. (11) becomes

$$
\left(\frac{d X}{d T}\right)^{2}=-\frac{2}{3} X^{3}-2 X T .
$$

The left and right-hand sides of Eq. (25) for the numerical solution $X(T)$ of Eq. (21) are plotted in Fig. 2. The solid black curve, showing the left-hand side $(d X / d T)^{2}$, is positive definite as one would expect. The dashed blue curve shows the right-hand side. If Eq. 25) was satisfied, the two curves would be identical and the right-hand side would be positive definite as well. This is clearly not the case.

What does this mean physically? According to Eq. (16), the dimensionless variable $T$ is simultaneously proportional to the proper time $\tilde{\tau}$, the energy $\chi$, and the angular momentum $\xi$. Values of $X(T)$ for which the right-hand side of Eq. 25) is negative correspond

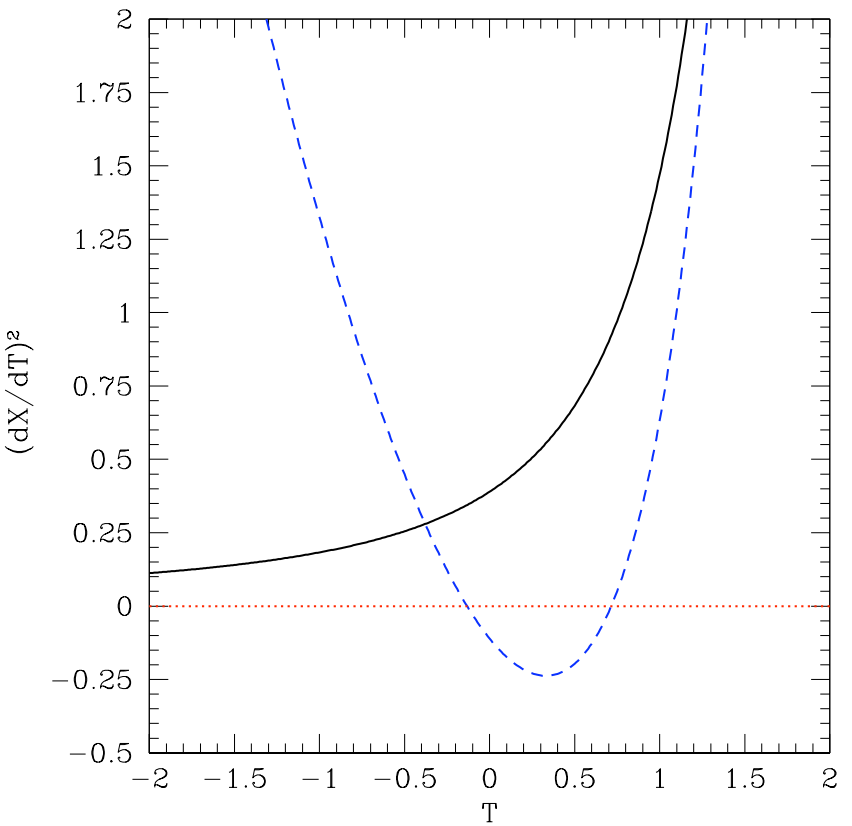

FIG. 2: The square of the dimensionless radial velocity $d X / d T$ as a function of dimensionless time $T$ during the transition from adiabatic inspiral to plunge. The solid black curve shows the left-hand side of Eq. 25) for the numerical solution to Eq. 21), while the dashed blue curve shows the right-hand side of Eq. 25) for this same numerical solution. The two curves clearly differ, and the dashed blue curve becomes negative when it falls below the dotted red line. Since $(d X / d T)^{2}$ is positive definite, this indicates that the numerical solution occupies a forbidden portion of $\{X, T\}$ parameter space.

to values of $\{\tilde{r}, \tilde{E}, \tilde{L}\}$ for which the effective potential $V(\tilde{r}, \tilde{E}, \tilde{L})$ exceeds the square of the energy $\tilde{E}^{2}$ in violation of Eq. (6). For example, at $T=0$ the energy and angular momentum equal their ISCO values, yet $X(0)>0$ as seen in Fig. 1. Since $V\left(\tilde{r}_{\mathrm{ISCO}}, \tilde{E}_{\mathrm{ISCO}}, \tilde{L}_{\mathrm{ISCO}}\right)=\tilde{E}_{\mathrm{ISCO}}^{2}$ and the effective potential $V$ is a monotonically increasing function of $X$ for $T=0$, the right-hand side of Eq. 25 must be negative at $T=0$ as seen in Fig. 2. The discrepancy between the left and right-hand sides of Eq. 225 is not a consequence of a breakdown in the Taylor expansion of the effective potential for $R, \xi \gtrsim 1$. Figure 2 shows that Eq. (25) is violated even for $X, T \simeq 1$, which corresponds to $R, \xi \ll 1$ when $\eta \ll 1$.

How can we reconcile the normalization of the 4velocity (4) and the geodesic equation (8) in the presence of radiation reaction? Although Eqs. (15) and (16) correctly specify the energy, angular momentum, and their derivatives on a circular orbit at $\tilde{r}_{\mathrm{ISCO}}$, there is no reason to expect them to hold exactly when the radial velocity is nonzero as it is during the transition. If we relax the requirement that $\chi=\xi$, we recover the third and fourth terms on the right-hand side of Eq. (11) that vanished in the previous treatment. We can retain the dimensionless 


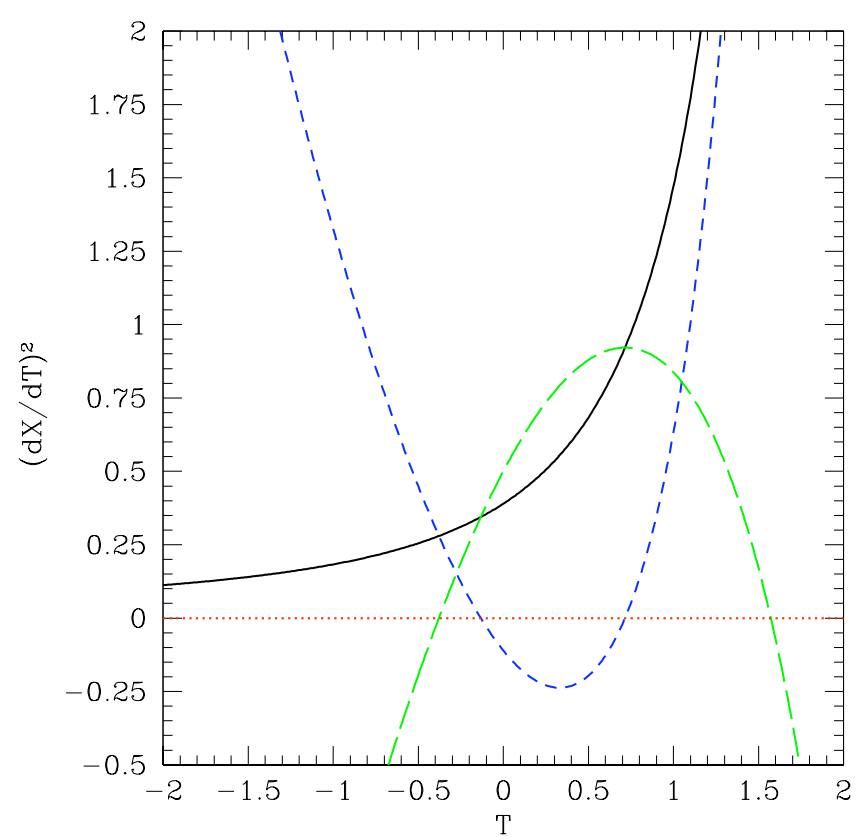

FIG. 3: The solid black, short-dashed blue, and dotted red curves in this plot are identical to those in Fig. 2, but we have also added the dimensionless difference between the energy and angular momentum $Y(T)$ which is given by the longdashed green curve. The short-dashed blue curve shows the first two terms on the right-hand side of Eq. 28, while the long-dashed green curve shows our new third term. Their sum equals the solid black curve, implying that Eq. 28 is now satisfied and the test particle's 4-velocity is properly normalized.

form of the equations of motion by defining

$$
\chi-\xi \equiv \eta^{6 / 5}(\chi-\xi)_{0} Y,
$$

where

$$
(\chi-\xi)_{0}=\alpha^{-4 / 5}(\beta \kappa)^{6 / 5}\left(\frac{\partial V}{\partial \tilde{L}}\right)^{-1} .
$$

The second-order equation (21) remains unchanged to lowest order in $\eta$, while Eq. 25) gains an additional term to become

$$
\left(\frac{d X}{d T}\right)^{2}=-\frac{2}{3} X^{3}-2 X T+Y
$$

Instead of Eq. 15], which would imply that $Y$ is constant throughout the transition, we evolve $Y$ according to

$$
\frac{d Y}{d T}=2 X .
$$

This is precisely what is required to restore the consistency between Eqs. (21) and (28) that exists for geodesic motion. Since Eq. (21) remains unchanged, its solution $X(T)$ remains unchanged as well.
We can solve Eq. 29] by inserting $X(T)$ into the righthand side and choosing the correct initial condition. This initial condition can be found by matching to the quasicircular inspiral at early times. During the inspiral, $\tilde{E}$ and $\tilde{L}$ are given by 11 .

$$
\begin{aligned}
\tilde{E}_{c}(\tilde{r}) & =\frac{1-2 / \tilde{r}+\tilde{a} / \tilde{r}^{3 / 2}}{\sqrt{1-3 / \tilde{r}+2 \tilde{a} / \tilde{r}^{3 / 2}}}, \\
\tilde{L}_{c}(\tilde{r}) & =\tilde{r}^{1 / 2} \frac{1-2 \tilde{a} / \tilde{r}^{3 / 2}+\tilde{a}^{2} / \tilde{r}^{2}}{\sqrt{1-3 / \tilde{r}+2 \tilde{a} / \tilde{r}^{3 / 2}}} .
\end{aligned}
$$

Taylor expanding about the ISCO,

$$
\begin{aligned}
\chi-\xi & \equiv \tilde{\Omega}_{\mathrm{ISCO}}^{-1}\left(\tilde{E}_{c}(r)-\tilde{E}_{\mathrm{ISCO}}\right)-\left(\tilde{L}_{c}(r)-\tilde{L}_{\mathrm{ISCO}}\right) \\
& \simeq \frac{1}{6}\left(\tilde{\Omega}^{-1} \frac{d^{3} \tilde{E}_{c}}{d \tilde{r}^{3}}-\frac{d^{3} \tilde{L}_{c}}{d \tilde{r}^{3}}\right)_{\mathrm{ISCO}} R^{3}
\end{aligned}
$$

Using the definitions of $X$ and $Y$ in Eqs. 19a and 26, this implies

$$
\begin{aligned}
Y & =\frac{1}{6} \alpha^{-1}\left(\tilde{\Omega}^{-1} \frac{d^{3} \tilde{E}_{c}}{d \tilde{r}^{3}}-\frac{d^{3} \tilde{L}_{c}}{d \tilde{r}^{3}}\right)_{\mathrm{ISCO}}\left(\frac{\partial V}{\partial \tilde{L}}\right)_{\mathrm{ISCO}} X^{3} \\
& =-\frac{4}{3} X^{3} \quad T \rightarrow-\infty
\end{aligned}
$$

Inserting Eqs. 22 and $(32)$ into the right-hand side of Eq. (28) shows that $d X / d T$ vanishes as $T \rightarrow-\infty$ as required by Eq. (22). The numerical solution $Y(T)$ with this initial condition is shown by the long-dashed green curve in Fig. 3. With the addition of this new term to the right-hand side, Eq. (28) is now satisfied ensuring that the 4-velocity is normalized according to Eq. 1a.

Our new term $Y(T)$ is important for more than just mathematical consistency. Equation (26) suggests that the physical quantity $\chi-\xi$ is proportional to $\eta^{6 / 5}$ during the transition, making it higher order than self-force corrections that scale as $\eta$. However, as the test particle plunges into the horizon, the asymptotic solution 23. and Eq. 29) imply

$$
\begin{aligned}
Y(T) & =Y(0)+\int_{0}^{T} \frac{d Y}{d T^{\prime}} d T^{\prime} \\
& \simeq Y(0)-\int_{0}^{T} \frac{12}{\left(T_{\text {plunge }}-T^{\prime}\right)^{2}} d T^{\prime} \\
& \simeq-\frac{12}{T_{\text {plunge }}-T}
\end{aligned}
$$

which diverges as $T \rightarrow T_{\text {plunge. }}$ This divergence can be seen from the limiting behavior of the three curves in Fig. 3. Although the solid black and short-dashed blue curves appear to converge as $T \rightarrow T_{\text {plunge }}$, their difference $Y(T)$ shown by the long-dashed green curve in fact diverges in accordance with Eq. (33). Does the divergence of the dimensionless $Y(T)$ imply a similar divergence in the physical quantity $\chi-\xi$ as the test particle plunges into the black hole? 
To answer this question, we must examine the validity of the asymptotic solution (23) as $T \rightarrow T_{\text {plunge }}$. Footnote 3 of OT notes that the divergence of $X(T)$ results from a breakdown in the dimensionless equation of motion (21) when higher-order terms in the Taylor expansion about the ISCO become important. This breakdown occurs at a coordinate radius $R_{\text {break }}$, which corresponds to a dimensionless radius $X_{\text {break }} \propto \eta^{-2 / 5}$ according to Eq. 19a. This in turn implies that $T_{\text {plunge }}-T_{\text {break }} \propto \eta^{1 / 5}$ by Eq. 23 and $Y_{\text {break }} \propto \eta^{-1 / 5}$ from Eq. (33). According to Eq. (26), the test particle's energy during the transition will receive a correction

$$
\Delta \tilde{E}_{\mathrm{norm}}=\eta^{6 / 5} \tilde{\Omega}_{\mathrm{ISCO}}(\chi-\xi)_{0} Y .
$$

When $Y \sim Y_{\text {break }}$, this correction will be linearly proportional to $\eta$ just like self-force corrections. It will be instructive to compare future self-force and time-domain perturbation theory calculations with this analytic result.

\section{MAXIMAL SPINS}

Kerr black holes [6] have spins $\tilde{a}<1$; objects with larger spins are "naked singularities" unclothed by an event horizon. Penrose 24 proposed that a cosmic censor protects general relativity by preventing the formation of such naked singularities, but this cosmic censorship conjecture has never been proven in full generality. Wald 25. determined that maximally spinning black holes could not accrete test particles that would drive them over the Kerr limit $\tilde{a}=1$, but Jacobson and Sotiriou 26] recently showed that black holes with spins $\delta \equiv 1-\tilde{a} \ll 1$ could be spun above this limit by accreting particles with a finite energy $E=\eta M \tilde{E}$. Barausse et al. 27] showed that gravitational radiation could not prevent some of these finite-mass-ratio mergers from producing a naked singularity, but that self-force corrections could be significant. Given that finite-mass-ratio effects may determine whether naked singularities can exist, it is worthwhile to examine what happens to the transition region in the maximally spinning limit.

The previous treatment of the transition from quasicircular inspiral to plunge assumes that the effective potential $V$ and fluxes $d \tilde{E} / d \tilde{\tau}$ and $d \tilde{L} / d \tilde{\tau}$ can be Taylor expanded in a neighborhood of the ISCO. However, as the black hole's spin approaches the Kerr limit $a \rightarrow M$, $r_{\mathrm{ISCO}}$ and the outer horizon $r_{+}$both approach $M$ in Boyer-Lindquist coordinates. This suggests that it may not be possible to construct a neighborhood of the ISCO that does not include the horizon. To determine whether or not such a neighborhood exists, we must compare $R_{+} \equiv \tilde{r}_{\text {ISCO }}-\tilde{r}_{+}$with $R_{0}$ in Eq. (20a) as $\delta \rightarrow 0$. In this limit [11,

$$
\begin{aligned}
\tilde{r}_{\mathrm{ISCO}} & \rightarrow 1+(4 \delta)^{1 / 3}, \\
\tilde{r}_{+} & \rightarrow 1+(2 \delta)^{1 / 2},
\end{aligned}
$$

implying $R_{+} \propto \delta^{1 / 3}$.

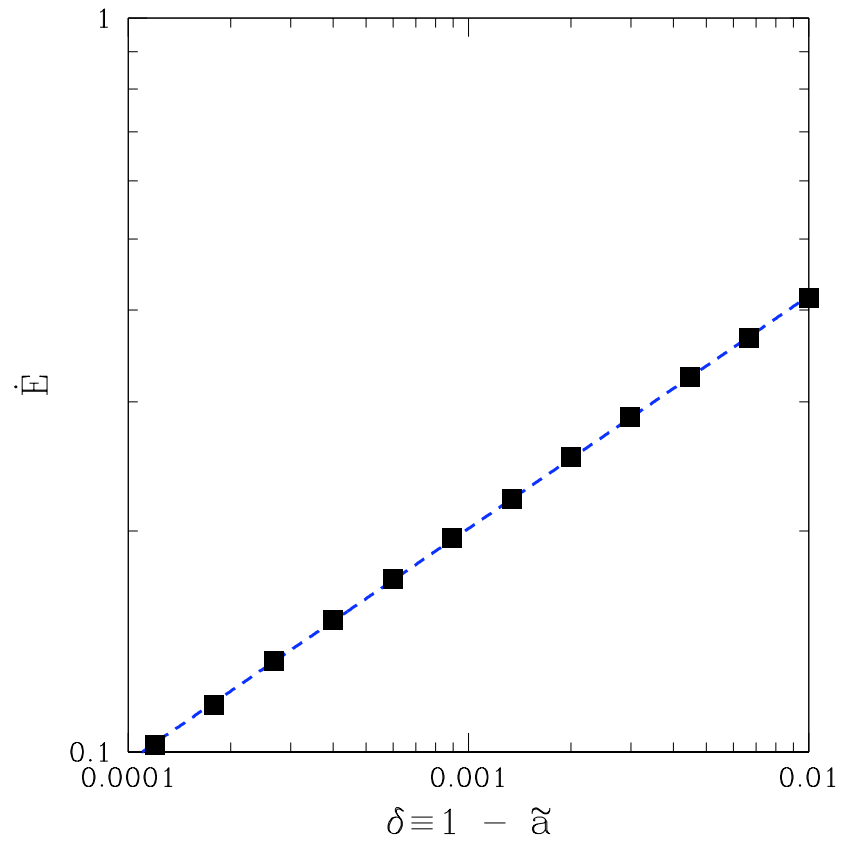

FIG. 4: The general-relativistic correction $\dot{\mathcal{E}}$ to the Newtonian quadrupole-moment formula for the GW luminosity as a function of black-hole spin $\delta \equiv 1-\tilde{a}$. The points have been calculated using the black-hole perturbation theory code GREMLIN [30] and fitted with a power law $\dot{\mathcal{E}}=A \delta^{m}$.

The behavior of $R_{0}$ as $\delta \rightarrow 0$ depends on the behavior of the fluxes $d \tilde{E} / d \tilde{\tau}$ and $d \tilde{L} / d \tilde{\tau}$. These in turn depend on $\dot{\mathcal{E}}$, defined by

$$
\dot{E}_{\mathrm{GW}}=\frac{32}{5} \eta^{2} \tilde{\Omega}^{10 / 3} \dot{\mathcal{E}}
$$

as the general-relativistic correction to the Newtonian quadrupole-moment formula for the GW luminosity [28]. The ISCO values of this correction $\dot{\mathcal{E}}$ were calculated for spins $-0.99 \leq \tilde{a} \leq 0.999$ in 28 , and have been calculated down to $\delta=10^{-4}$ [29] using the GREMLIN (Gravitational Radiation in the Extreme Mass Ratio Limit) code presented in 30. We have fitted these calculated values to a power law $\dot{\mathcal{E}}=A \delta^{m}$ as shown in Fig. 4. The best-fit parameters for this power law are $A=1.80, m=0.317$. The summation over spheroidal harmonics of the Weyl scalar $\psi_{4}$ needed to compute $\dot{\mathcal{E}}$ converges very slowly in the limit $\delta \rightarrow 0$, so our best-fit parameters should be regarded with caution until they can be confirmed by a technique better suited to this limit. Chrzanowski 31 estimated that

$$
\dot{E}_{\mathrm{GW}} \sim \eta^{2} R_{+},
$$

which also suggests that $m \sim 1 / 3$. The rough agreement between our numerical fit and Chrzanowski's estimate gives us some confidence that $m \simeq 1 / 3$ is close to the correct value. 
The scale $R_{0}$ of the transition region depends on the relativistic correction $\dot{\mathcal{E}}$ through $\kappa$, which was defined in Eq. 177) and can be expressed as

$$
\kappa=\frac{32}{5}\left(\tilde{\Omega}^{7 / 3} \frac{d \tilde{t}}{d \tilde{\mathcal{E}}} \dot{\mathcal{E}}\right)_{\mathrm{ISCO}} .
$$

For circular equatorial Kerr geodesics, as $\delta \rightarrow 0$,

$$
\begin{aligned}
\tilde{\Omega} & \rightarrow \frac{1}{2}\left[1-\frac{3}{4}(4 \delta)^{1 / 3}\right], \\
\frac{d \tilde{t}}{d \tilde{\tau}} & \rightarrow \frac{4}{\sqrt{3}}(4 \delta)^{-1 / 3}, \\
\kappa & \rightarrow \frac{16}{5 \sqrt{3}} A \delta^{m-1 / 3}, \\
\alpha & \rightarrow 1 \\
\beta & \rightarrow \frac{\sqrt{3}}{2}(4 \delta)^{1 / 3}, \\
\frac{\partial V}{\partial \tilde{L}} & \rightarrow \frac{4}{\sqrt{3}}(4 \delta)^{1 / 3},
\end{aligned}
$$

implying

$$
\begin{aligned}
R_{0} & \propto \delta^{2 m / 5} \\
\tau_{0} & \propto \delta^{-m / 5} \\
(\chi-\xi)_{0} & \propto \delta^{6 m / 5-1 / 3}
\end{aligned}
$$

from the definitions in Eqs. 201 and (27).

According to Eq. (19a), the transition region will be cut off by the horizon at a dimensionless radius

$$
X_{+}=-\eta^{-2 / 5} \frac{R_{+}}{R_{0}} \propto-\eta^{-2 / 5} \delta^{1 / 3-2 m / 5}
$$

as $\delta \rightarrow 0$. We plot $X_{+}(\delta)$ for several mass ratios and our best-fit value of $m$ in Fig. 5 . Since $m<5 / 6, X_{+} \rightarrow 0$ in the limit of large spins. One should not expect GW emission beyond $X_{+}$, so Eq. (24) overestimates the additional energy and angular momentum radiated during the transition. In the limit of large spins, $\Delta \tilde{E}_{\mathrm{tr}}$ and $\Delta \tilde{L}_{\mathrm{tr}}$ will be suppressed by a factor $T_{0} / T_{\text {plunge }} \simeq 0.21$, where $X\left(T_{0}\right)=0$.

Despite this suppression, the extra energy and angular momentum radiated during the transition remain proportional to $\kappa \tau_{0} \propto \delta^{4 m / 5-1 / 3}$, which diverges for $m<5 / 12$. Our numerical fit shown in Fig. 4 suggests that $m$ satisfies this inequality. However, this divergence might not be physical; it could merely reflect a breakdown of the Taylor expansion of the equation of motion given in Eq. (14). The first two terms on the right-hand side of this equation are proportional to $\eta^{4 / 5}$, while Eq. (26) shows that the third term is proportional to $\eta^{6 / 5}$. This higher-order dependence on $\eta$ justifies neglecting this third term for modest spins, but we must also consider how each term depends on $\delta$ in the maximally spinning limit. In this limit, the first two terms are proportional to $\delta^{4 m / 5}$ according to Eqs. (39) and (40) while the third term is

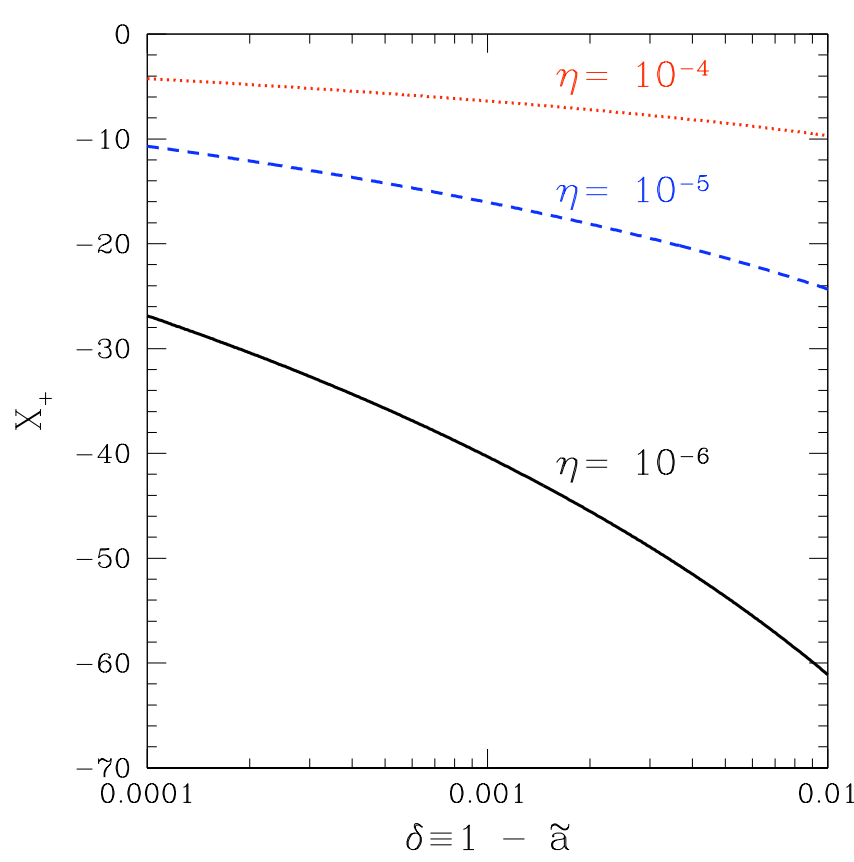

FIG. 5: The dimensionless horizon radius $X_{+}=$ $-\eta^{-2 / 5} R_{+} / R_{0}$ as a function of $\delta \equiv 1-\tilde{a}$. The dotted red, dashed blue, and solid black curves correspond to mass ratios $\eta=10^{-4}, 10^{-5}$, and $10^{-6}$ respectively. $X_{+} \rightarrow 0$ as $\delta \rightarrow 0$ because the relativistic correction $\dot{\mathcal{E}}$ to the energy radiated in GWs has a power-law index $m<5 / 6$.

proportional to $\delta^{6 m / 5-1 / 3}$. For $m<5 / 6$, this third term will dominate over the first two terms, indicating a breakdown of the original dimensionless equation of motion 211. For completeness, we note that we have neglected an additional term

$$
-\frac{1}{12}\left(\frac{\partial^{4} V}{\partial \tilde{r}^{4}}\right)_{\mathrm{ISCO}} R^{3}
$$

at $\mathcal{O}\left(\eta^{6 / 5}\right)$ on the right-hand side Eq. 14. This term is proportional to $\delta^{6 m / 5}$ as $\delta \rightarrow 0$ and thus can always be neglected.

Including the third term in Eq. (14) on the right-hand side of Eq. 21) yields

$$
\frac{d^{2} X}{d T^{2}}=-X^{2}-T+\eta^{2 / 5} C Y
$$

where

$$
C \equiv-\frac{1}{2} \alpha^{-3 / 5}(\beta \kappa)^{2 / 5} \tilde{\Omega} \frac{\partial^{2} V}{\partial \tilde{E} \partial \tilde{r}}\left(\frac{\partial V}{\partial \tilde{L}}\right)^{-1} \propto \delta^{2 m / 5-1 / 3}
$$

diverges as $\delta \rightarrow 0$ for $m<5 / 6$. Y must evolve according to

$$
\frac{d Y}{d T}=2 X+2 \eta^{2 / 5} C Y \frac{d X}{d T}
$$




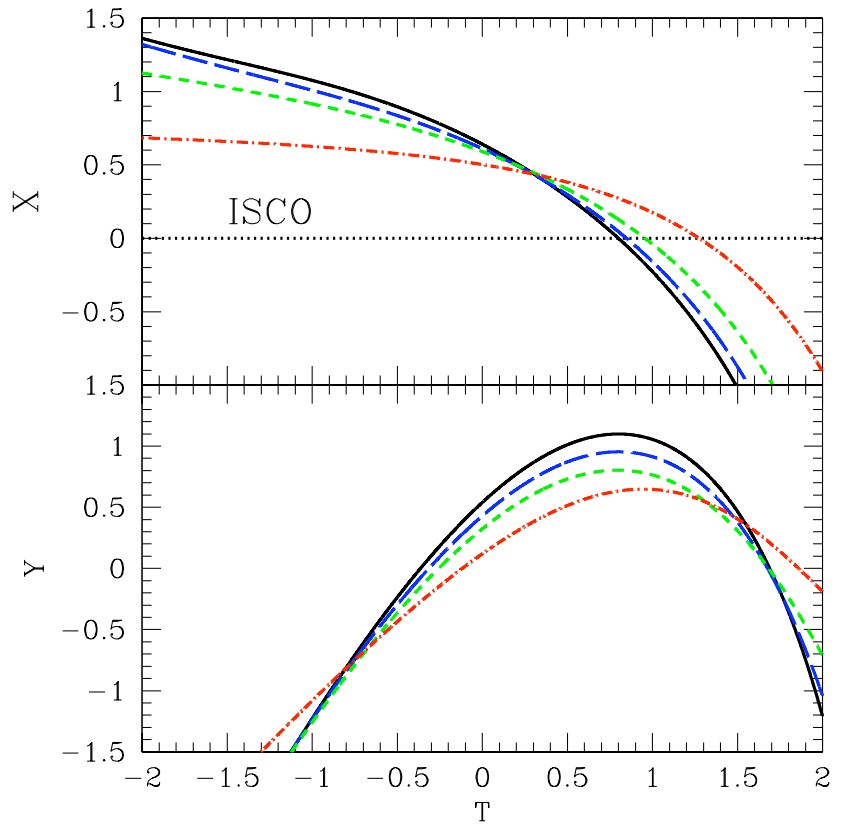

FIG. 6: Upper panel: The dimensionless radius $X$ as a function of dimensionless time $T$ in the limit $\delta \equiv 1-\tilde{a} \rightarrow 0$. The solid black curve is the OT solution, while the long-dashed blue, short-dashed green, and dot-dashed red curves show our solutions with $\eta=10^{-3}$ and $\delta=10^{-2}, 10^{-4}$ and $10^{-6}$ respectively. Lower panel: The dimensionless difference $Y$ between the energy and angular momentum for the solutions shown in the upper panel.

to preserve the normalization the 4 -velocity given by Eq. 28). The product $\eta^{2 / 5} C$ thus measures the deviation of $X(T)$ from the OT solution in the limit of large spins. We plot our new solutions $X\left(T, \eta^{2 / 5} C\right)$ for a mass ratio $\eta=10^{-3}$ and spins $\delta=10^{-2}, 10^{-4}$ and $10^{-6}$ in Fig. 6. Although our solutions diverge from the OT solution as $\eta^{2 / 5} C$ increases, the test particle crosses the ISCO $X=0$ (shown by the horizontal dotted line) at a later dimensionless time $T$. This implies that more energy and angular momentum are radiated and the divergence in $\Delta \tilde{E}_{\mathrm{tr}}$ and $\Delta \tilde{L}_{\mathrm{tr}}$ as $\delta \rightarrow 0$ cannot be avoided. One must look to finite-mass ratio effects beyond the scope of this paper to eliminate this unphysical result.

One such effect that might provide a solution to this problem is the spinning down of the black hole due to the extraction of angular momentum by the superradiant scattering of GWs earlier in the inspiral 23. This effect implies that even an initially maximally spinning black hole will have $\delta \propto \eta$ by the time the test particle reaches the transition region. The additional energy and angular momentum $\Delta \tilde{E}_{\mathrm{tr}}, \tilde{L}_{\mathrm{tr}} \propto \eta^{4 / 5} \delta^{4 m / 5-1 / 3} \propto \eta^{4 m / 5+7 / 15}$ would then remain finite even if $\delta=0$ initially.

How does the energy and angular momentum radiated during the transition affect the spin of the final black hole produced in the merger? If we assume that energy and angular momentum are conserved after the end of the transition, the final spin will be

$$
\begin{aligned}
\tilde{a}_{f} & =\frac{\tilde{a}+\eta\left(\tilde{L}_{\mathrm{ISCO}}-\Delta \tilde{L}_{\mathrm{tr}}\right)}{\left[1+\eta\left(\tilde{E}_{\mathrm{ISCO}}-\Delta \tilde{E}_{\mathrm{tr}}+\Delta \tilde{E}_{\mathrm{norm}}\right)\right]^{2}} \\
& \simeq \tilde{a}+\eta\left(\Delta \tilde{a}_{\mathrm{ISCO}}+\Delta \tilde{a}_{\mathrm{tr}}+\Delta \tilde{a}_{\mathrm{norm}}\right)
\end{aligned}
$$

for $\eta \ll 1$, where in the limit $\delta \rightarrow 0$

$$
\begin{aligned}
\Delta \tilde{a}_{\mathrm{ISCO}} & \equiv \tilde{L}_{\mathrm{ISCO}}-2 \tilde{E}_{\mathrm{ISCO}} \rightarrow \frac{\sqrt{3}}{2}(4 \delta)^{2 / 3} \\
\Delta \tilde{a}_{\mathrm{tr}} & \equiv-\left(1-2 \tilde{\Omega}_{\mathrm{ISCO}}\right) \Delta \tilde{L}_{\mathrm{tr}} \propto \eta^{4 / 5} \delta^{4 m / 5}, \\
\Delta \tilde{a}_{\mathrm{norm}} & \equiv-2 \Delta \tilde{E}_{\mathrm{norm}} \propto \eta^{6 / 5} \delta^{6 m / 5-1 / 3}
\end{aligned}
$$

Although our new correction $\Delta \tilde{a}_{\text {norm }}$ to the black hole's final spin is subdominant in $\eta$, for $m<5 / 6$ it becomes the dominant correction as $\delta \rightarrow 0$. Since $\Delta \tilde{E}_{\text {norm }}>0$ when the particle crosses the horizon in the limit $\delta \rightarrow$ 0 , our analysis suggests that gravitational radiation in the transition region will not promote the formation of naked singularities. This supports our earlier result in 23, where we showed that the superradiant scattering of GWs emitted at $\tilde{r}>\tilde{r}_{\text {ISCO }}$ would also reduce the final spin for $\tilde{a} \gtrsim 0.998$.

\section{DISCUSSION}

The existence of an ISCO is one of the most distinctive features of a black hole. Although the presence of an ISCO indirectly affects the luminosity and spectra of accreting black holes, it is more cleanly probed by the GWs emitted as test particles (compact objects like white dwarfs, neutron stars, and stellar-mass black holes) plunge into supermassive black holes. Such GWs are a primary source for the proposed space-based GW detector LISA. The LISA detection strategy relies on convolving observations with theoretically determined GW templates. Understanding how a test particle's position, energy, and angular momentum evolve near the ISCO is an important first step toward constructing these templates.

Previous studies [12, 13, identified a transition region near the ISCO where neither the quasicircular approximation nor the assumption of geodesic motion are valid. They solved the radial equation of motion in this region by Taylor expanding it about the ISCO. Ori and Thorne (2000) [12 fixed the energy and angular momentum fluxes to their ISCO values, while Buonanno and Damour (2000) [13] worked with the Hamilton equations of motion and did not need to specify an explicit energy flux. The dimensionless solution $X(T)$ obtained by both groups can be rescaled to determine the evolution of the test particle's position, energy, and angular momentum for arbitrary mass ratios and spins. The simplicity of their approach and the universality of their solution are highly appealing, but a closer examination reveals that 
their solution does not properly normalize the particle's 4-momentum.

We undertook this study to see whether this technical problem could be easily remedied, or was a symptom of a more serious flaw in their approach. We found that by introducing a correction $\Delta \tilde{E}_{\text {norm }}$ to the particle's energy at a higher order in the mass ratio $\eta \ll 1$, we could properly normalize the 4-momentum without altering their universal solution $X(T)$ at lowest order. This relatively modest correction increases our confidence in this particle trajectory, and validates its use as a source for the construction of GW waveforms as in [15.

In addition to its role in constructing GW templates, gravitational radiation during the transition also affects whether a test-particle merger can produce a naked singularity by increasing a black hole's spin above the Kerr limit $\tilde{a}=1$. Our calculation of the energy and angular momentum flux at the ISCO as $\delta \equiv 1-\tilde{a} \rightarrow 0$ suggests that the total energy $\Delta \tilde{E}_{\mathrm{tr}}$ and angular momentum $\Delta \tilde{L}_{\text {tr }}$ radiated during the transition diverge in this limit. This divergence cannot be physical, and must therefore be moderated by high-spin corrections beyond the scope of this paper. Despite this divergence, the change $\Delta \tilde{a}_{\mathrm{tr}}$ in the black hole's spin due to this radiation remains finite in magnitude and negative in sign. It therefore reduces the likelihood that a naked singularity will be produced, as does our new correction $\Delta \tilde{a}_{\text {norm }}$ which is also negative and becomes dominant as $\delta \rightarrow 0$.

Throughout this paper, we have neglected self-force corrections to the particle's energy even though they are formally lower order in $\eta$ than our correction $\Delta \tilde{E}_{\text {norm }} \propto$ $\eta^{6 / 5}$. Far from the ISCO, where $|Y| \gg 1$, our correction should dominate self-force effects despite its scaling with $\eta$. Near the ISCO however, self-force corrections should be significant. Reliable calculations of the selfforce do not yet exist near the ISCOs of highly spinning black holes. Comparing such self-force calculations with our analysis as they become available is a subject for future work. Another important future test of our analysis would be to use time-domain perturbation theory to calculate the energy and angular momentum radiated by a particle whose position is given by an appropriate rescaling of the solution $X(T)$. Agreement with our predicted solution $Y(T)$ would strongly support our conjecture that proper normalization of the 4-momentum can be used to predict GW emission. Comparison with numerical relativity may soon be possible as well, since simulations with mass ratios as small as $\eta=0.01$ have recently been performed 32 . Our predictions could be even further improved by including the gravitational radiation emitted near the horizon [33]. We look forward to comparing our calculation to these alternative theoretical approaches in the near future, and comparing all these predictions to GW observations in the hopefully not too distant future.

Acknowledgements. I would like to thank Sterl Phinney for his advice during the initial stages of this project, and Scott Hughes for insight into the emission of gravitational radiation in the limit of large spins. Jeandrew Brink, Marc Favata, Chris Hirata, Guglielmo Lockhart, Samaya Nissanke, and David Tsang also offered helpful comments on this work.
[1] J. Kormendy and D. Richstone, Ann. Rev. Astron. Astrophys. 33, 581 (1995).

[2] J. N. Bahcall and R. A. Wolf, Astrophys. J. 209, 214 (1976).

[3] J. Frank and M. J. Rees, Mon. Not. Roy. Astron. Soc. 176, 633 (1976).

[4] S. Sigurdsson and M. J. Rees, Mon. Not. Roy. Astron. Soc. 284, 318 (1997).

[5] LISA, http://lisa.nasa.gov/.

[6] R. P. Kerr, Phys. Rev. Lett. 11, 237 (1963).

[7] B. Carter, Phys. Rev. 174, 1559 (1968).

[8] M. Walker and R. Penrose, Commun. Math. Phys. 18, 265 (1970).

[9] S. A. Teukolsky, Astrophys. J. 185, 635 (1973).

[10] P. C. Peters and J. Mathews, Phys. Rev. 131, 435 (1963).

[11] J. M. Bardeen, W. H. Press and S. A. Teukolsky, Astrophys. J. 178, 347 (1972).

[12] A. Ori and K. S. Thorne, Phys. Rev. D 62, 124022 (2000).

[13] A. Buonanno and T. Damour, Phys. Rev. D 62, 064015 (2000).

[14] P. A. Sundararajan, Phys. Rev. D 77, 124050 (2008).

[15] P. A. Sundararajan, G. Khanna and S. A. Hughes, Phys. Rev. D 81, 104009 (2010).

[16] R. H. Boyer and R. W. Lindquist, J. Math. Phys. 8, 265 (1967).

[17] Y. Mino, M. Sasaki and T. Tanaka, Phys. Rev. D 55,
3457 (1997).

[18] E. Poisson, Living Rev. Relativity 7, 6 (2004) http://www.livingreviews.org/lrr-2004-6.

[19] L. Barack, Class. Quant. Grav. 26, 213001 (2009).

[20] S. A. Hughes and R. D. Blandford, Astrophys. J. 585, L101 (2003).

[21] A. Buonanno, L. E. Kidder and L. Lehner, Phys. Rev. D 77, 026004 (2008).

[22] M. Kesden, Phys. Rev. D 78, 084030 (2008).

[23] M. Kesden, G. Lockhart, E. S. Phinney, Phys. Rev. D82, 124045 (2010).

[24] R. Penrose, Riv. Nuovo Cim. 1, 252 (1969), reprinted in [Gen. Rel. Grav. 34, 1141 (2002)].

[25] R.M. Wald, Ann. Phys. 82, 548 (1974).

[26] T. Jacobson, T. P. Sotiriou, Phys. Rev. Lett. 103, 141101 (2009).

[27] E. Barausse, V. Cardoso, G. Khanna, Phys. Rev. Lett. 105, 261102 (2010).

[28] L. S. Finn, K. S. Thorne, Phys. Rev. D62, 124021 (2000).

[29] S. A. Hughes, private communication.

[30] S. A. Hughes, Phys. Rev. D61, 084004 (2000).

[31] P. L. Chrzanowski, Phys. Rev. D13, 806-818 (1976).

[32] C. O. Lousto, Y. Zlochower, Phys. Rev. Lett. 106, 041101 (2011).

[33] Y. Mino, J. Brink, Phys. Rev. D78, 124015 (2008). 